Canadian University Music Review

Revue de musique des universités canadiennes

\title{
Marcelle Guertin. De la lecture à l'audition d'un texte musical : une étude des thèmes dans le Livre I des Préludes pour piano de Debussy. Montréal : Presses de l’Université de Montréal, collection « Sémiologie et analyse musicales », 1990, 245 pp.
}

\section{Marie-Thérèse Lefebvre}

Volume 11, numéro 2, 1991

URI : https://id.erudit.org/iderudit/1014205ar

DOI : https://doi.org/10.7202/1014205ar

Aller au sommaire du numéro

\section{Éditeur(s)}

Canadian University Music Society / Société de musique des universités canadiennes

ISSN

0710-0353 (imprimé)

2291-2436 (numérique)

Découvrir la revue

Citer ce compte rendu

Lefebvre, M.-T. (1991). Compte rendu de [Marcelle Guertin. De la lecture à l'audition d'un texte musical : une étude des thèmes dans le Livre I des Préludes pour piano de Debussy. Montréal : Presses de l’Université de Montréal, collection " Sémiologie et analyse musicales », 1990, 245 pp.] Canadian University Music Review / Revue de musique des universités canadiennes, 11(2), 154-157. https://doi.org/10.7202/1014205ar

(c) Canadian University Music Society / Société de musique des universités canadiennes, 1991
Ce document est protégé par la loi sur le droit d'auteur. L’utilisation des services d'Érudit (y compris la reproduction) est assujettie à sa politique d'utilisation que vous pouvez consulter en ligne.

https://apropos.erudit.org/fr/usagers/politique-dutilisation/ 
"The Fisher years: The Toronto Conservatory of Music, 1886-1913," is the first publication from her large research project on the history of The Royal Conservatory of Music (originally the Toronto Conservatory of Music). This is a fascinating study in that, for the first time, light is shed on the rather tangled background of one of Canada's pre-eminent music institutions. Jones's work is based on extensive archival research, and the article is documented fully with references to heretofore unpublished Conservatory records as well as with some engaging photographs, courtesy of the University of Toronto archives. Of particular interest is the author's discussion of the contributions and personalities of Edward Fisher and F.H. Torrington and of the background of the examination system which remains the backbone of the RCM. The third article in CanMus 4 is a detailed chronicle by Colin Eatock of the contemporary music group known since 1982 as Arraymusic. Eatock examines the group's original goals, financial problems, participants (composers and performers), concert activity, and changing motivations and directions. Perhaps most important is the group's dedication to contemporary Canadian music. As Eatock suggests, this dedication and much hard work have made Arraymusic a vital part of the music scene in Toronto.

CanMus Documents Volumes 1, 2, and 4 represent an important contribution to musical Canadiana. The range of subject areas covered in these volumes conference proceedings on historical topics and contemporary issues to scholarly studies - demonstrates the diversity and the commitment of the Institute for Canadian Music.

Gordon E. Smith

MARCELLE GUERTIN. De la lecture à l'audition d'un texte musical : une étude des thèmes dans le Livre I des Préludes pour piano de Debussy. Montréal: Presses de l'Université de Montréal, collection «Sémiologie et analyse musicales », 1990, $245 \mathrm{pp}$.

Pour ceux et celles qui ont suivi le développement de la théorie d'analyse sémiologique proposée par Jean-Jacques Nattiez, en 1975, dans Fondements d' une sémiologie de la musique ${ }^{1}$, le livre de Marcelle Guertin constitue le guide pédagogique essentiel à l'application de cette théorie dans ses volets «neutre » et « esthésique ».

1 Paris : Union Générale d'Éditions, coll. « 10/18 ». 
Malgré les quelques réticences que j'éprouve à l'égard du niveau « neutre », je demeure éblouie par la clarté et la limpidité du discours écrit utilisé pour la description non seulement des résultats d'une telle analyse, mais également - et surtout - des processus par lesquels l'auteur y est arrivée. On trouve en effet dans ce travail, que je qualifie de " guide méthodologique de l'apprenti-sémiologue », l'essentiel des définitions des concepts qui sont à la base de cet outil d'analyse (division tripartite, pp.16-17; syntagme,p. 27 ;paradigme,p. 31 ; paradigme des paradigmes, p. 74 ; fonction et distribution des unités, p. 74), définitions qui permettent au lecteur de mieux saisir le cheminement de l'auteure : la découverte de constantes stylistiques que lui révèle l'étude du corpus choisi, après une longue traversée du désert. «Nous avons longtemps erré à travers nos paradigmes avant qu'une clé formelle ne nous autorise à pénétrer dans l'édifice » écrit-elle p. 68, nous rappelant ainsi la fragilité et l'incertitude des voies de la recherche.

L'introduction nous situe d'emblée dans la problématique sémiologique et chaque étape de la réflexion est décrite avec précision, comme si l'auteure prévoyait à chaque phrase les questions de ses lecteurs ; son sens de la communication et son expérience pédagogique entrent ici en jeu. S'appuyant sur la tripartition préconisée par Nattiez, elle apporte des nuances importantes sur ce fameux niveau « neutre » auquel elle donnera, dans son travail, deux directions : le neutre proprement dit, soit l'oeuvre-partition (de caractère statique), et le " neutre orienté vers l'esthésique », soit l'œuvre-projection sonore (de caractère dynamique), telle que filtrée et rendue par l'interprète. Ces deux directions occuperont respectivement et successivement une tranche de l'ouvrage, le but principal de l'auteure étant le suivant : montrer, en prenant appui sur un corpus précis de mélodies (ou thèmes) de Debussy, comment l'analyse visuelle des signes d'une partition conduit à des résultats qui ne coïncident pas toujours avec l'analyse des données sonores du même texte musical. La démonstration se fera donc en deux temps, l'auteure se penchant d'abord exclusivement (ou presque) sur le texte imprimé et dégageant, à l'aide d'une méthode très explicite, une série de règles de fonctionnement du corpus retenu; après quoi - deuxième partie de l'ouvrage -, la méthode analytique jusque-là utilisée est remise en cause par l'écoute. Examinons d'un peu plus près de quoi il retourne.

Quatre chapitres composent la première partie. Le chapitre initial consiste en un panorama général de la démarche. L'auteure y présente l'objet de son étude : chacun des thèmes figurant dans les douze Préludes (44 thèmes au total), dont il s'agit " d'interroger la facture mélodique considérée en et pour elle-même, comme événement autonome et détaché de tout ce qui l'accompagne et l'environne " (p. 23), donc comme strict mode d'élaboration des hauteurs et intervalles, en ignorant l'harmonie (p. 24), le rythme, les intensités, les attaques, 
le phrasé(p. 28), et même les silences (p. 33).L'objet est vraiment microscopique, mais l'auteure s'en explique parfaitement, exemples à l'appui. Elle signale que l'examen de cet objet se situera au niveau neutre "sans se préoccuper, provisoirement, de la validité poïétique ou esthésique des manipulations et résultats ", mais ajoute, dans un même élan, que cette lecture des signes de la partition tient compte de la perception auditive de l'analyste, « qui se joue, se chante et prête constamment l'oreille aux êtres musicaux qu'il observe » (p. 24). Donc, pas si neutre ce «neutre », finalement, qui nous renvoie à la question : estce qu'un objet musical peut exister hors du champ de la perception auditive? (Oui si, comme Nattiez, on croit aux universaux et à l'existence d'un modèle dans l'univers platonicien des idées.)

Ayant apporté ces précisions, l'auteure présente ensuite les quatre étapes sur lesquelles repose son enquête de niveau neutre : constitution, au sein du corpus entier, d'un mini-corpus expérimental; segmentation des énoncés de ce minicorpus sur la base sur la notion de répétition et dont l'analyse sera fondamentale pour la crédibilité de ce travail; caractérisation stylistique du mini-corpus; et, finalement, application de ces conclusions au corpus entier.

Ainsi, si ce panorama de la démarche explicitée dans le chapitre premier est bien compris, le reste se lit comme un roman, ou presque ! La leçon d'anatomie peut commencer, la musique disparaît dans le deuxième chapitre au profit de la méthode. L'auteure entame alors la mise en paradigme des énoncés, " pour faire apparaître dans chaque thème un mode d'élaboration mélodique » (p.38) et pour créer de toutes pièces un objet d'analyse de niveau neutre dont elle tire trois catégories : thèmes à segmentation unique, à deux niveaux de segmentation et doublement ou triplement segmentables à un même niveau.

La façon dont chaque étape est décrite et dont chaque choix est fait nous donne l'impression de plonger au coeur même de la réflexion du chercheur et de ses questionnements, comme en atteste l'argumentation sur la possibilité d'attribuer au premier thème du Prélude VI, « ... Des pas sur la neige », deux délimitations différentes.

Dans les deux chapitres suivants, consacrés à la caractérisation stylistique du mini-corpus puis du corpus entier, $l$ 'auteure nous révèle l'aspect novateur de son approche : les constantes ou règles qui se dégagent de la construction d'un métaparadigme (p. 74), constantes qu'elle transpose dans une écriture par symboles (énumérés aux pp.78-82) en se justifiant ainsi : « Plus on admet des segments dans un champ de comparaison, plus on tend à oblitérer l'information locale pour mettre l'accent sur des propriétés plus abstraites » (p. 75). 
Elle répond à l'exigence du critère de validité de la démarche sémiologique à travers l'élaboration d'un modèle fondamental et de ses variantes, ce qui la contraint à utiliser une catégorie portant le nom - « d'un strict point de vue analytique ", prévient-elle (p. 100) - de " déchets » ou " unité à fonction non définie " (apax) par laquelle l'objet d'analyse se départit des objets musicaux hétérogènes (ceux qui marquent la différence, dirions-nous), " afin d'éviter d'encombrer l'enquête stylistique d'un foisonnement de données susceptibles de provoquer la dispersion de l'information plutôt que sa concentration »(p. 96).

Sans vouloir ouvrir un débat, on peut se demander si ce n'est pas justement à ces objets hétérogènes que réfère Boulez, lorsqu'il analyse le nouveau processus mental de mise en forme et de développement d'une oeuvre du $\mathrm{XX}^{\mathrm{e}}$ siècle : « La pensée musicale, écrit-il, agirait désormais sur des entités non réalisées au départ de l'oeuvre et ferait en sorte de découvrir en cours de route les différents aspects du possible, aspects impliqués naturellement au départ mais non ouvertement exprimés $»^{2}$.

La deuxième partie du travail nous ramène à l'objet musical. L'auteure nous invite à une lecture-audition des thèmes et propose une amorce de méthode analytique basée sur l'écoute, définissant d'abord l'auditeur cible auquel elle s'adresse : celui qui est familier avec l'objet musical à étudier, qui connaît les concepts sur lesquels s'appuira l'analyse et qui, en troisième lieu, a l'expérience de ce genre d'audition - en l'occurrence, l'auteure elle-même, qui sert de " cobaye » à la démonstration (p. 136). L'objectif consiste ici à re-délimiter cet objet nommé " thème » (avec, cette fois, tout ce qui l'accompagne et l'environne dans les pièces) et à apporter un « point d'écoute » critique à l'enquête préalablement faite au niveau neutre. L'approche permet, entre autres, de relever certaines difficultés de segmentation en situation d'écoute, difficultés provoquées tantôt par l'action conflictuelle des différents paramètres entre eux, tantôt par des irrégularités rythmiques - non indiquées dans la partition mais pourtant bel et bien perçues à l'audition (p. 155). On saisit ici combien l'objet musical est beaucoup plus difficile à cerner que l'objet d'analyse purifié de tous ses « apax » ...

Marie-Thérèse Lefebvre

2 Pierre Boulez, « Nécessité d'une orientation esthétique II 》 (1963), Revue de musique des universités canadiennes, 7, 1986, pp. 68-69. 